\title{
Gauge Potentials and Bundles Over the 4-Torus
}

\author{
C. Nash
}

Department of Mathematical Physics, St. Patrick's College, Maynooth, Ireland

\begin{abstract}
The construction of principal bundles over a four dimensional torus is considered. The class of groups considered is $\mathrm{SU}(n) / Z_{n}$, and for this class the Pontrjagin class has even integer values.
\end{abstract}

\section{Introduction}

This paper considers principal fibre bundles over a four-dimensional torus. Physically a four-dimensional torus corresponds to space-time being a kind of Euclidean box with periodic boundary conditions. Fibre bundles enter when one considers non-Abelian gauge fields inside this box. This physical picture has been considered by a number of people, cf., for example, [1] and references cited therein.

In [1] it is argued that the gauge groups $\mathrm{SU}(n) / Z_{n}, n=2,3, \ldots$ are physically important $\left(Z_{n}\right.$ stands for the centre of the group $\mathrm{SU}(n)$, hence for each $n, Z_{n}$ is isomorphic to the $n^{\text {th }}$ roots of unity). The topology of space-time is $S^{1} \times S^{1} \times$ $S^{1} \times S^{1}$, where $S^{1}$ is a unit circle. We shall denote space-time by $T^{4}$. Underlying the non-Abelian gauge field is a fibre bundle and so we are led to the construction of all $\mathrm{SU}(n) / Z_{n}$ bundles over $T^{4}$. We describe, in what follows, a method for carrying out this construction. In Sect. 2 we treat the case $n=2$, and in Sect. 3 the case $n>2$. An important mathematical tool in the calculations will be the generalised cohomology theory known as $K$-theory.

\section{The $n=2$ Case}

When $n=2$ there is the well known result, of a kind typical for Lie groups of low dimension, that $\mathrm{SU}(2) / Z_{2} \simeq \mathrm{SO}(3)$. Thus we wish to construct all $\mathrm{SO}(3)$-bundles over $T^{4}$. In contrast to the case where the base space is a sphere $S^{k}$ the calculation is not completely straightforward. It turns out to be most easily accomplished by resorting to a well known mathematical tool of bundle theory known as $K$-theory. $K$-theory is a kind of generalised cohomology theory defined for vector bundles. For an introduction to $K$-theory, cf. the works cited in [2]. The $K$-theory for $T^{4}$ considers all vector bundles $E$ over $T^{4}$ and assembles them together into equivalence classes - two bundles $E$ and $F$ are equivalent if the addition of a trivial 
bundle $I^{j}$ to each of them renders them isomorphic [2]: $E \oplus I^{j} \simeq F \oplus I^{k}$. Although the $K$-theory over $T^{4}$ considers all vector bundles over $T^{4}$ of all possible ranks, we shall nevertheless be able to pin down those bundles with $\mathrm{SO}(3)$ as their structure group and identify their corresponding principal bundles. We shall use the notation of Husemoller [2]. Since $\mathrm{SO}(3)$ is an orthogonal group, the corresponding $K$-theory is denoted by $\widetilde{K} O$-the so-called reduced real $K$-theory [2]. In general $\widetilde{K} O(M)$ for some $M$ forms a ring, with multiplication and addition provided by tensor product and direct sum respectively. If $M=T^{4}$, then $\tilde{K} O\left(T^{4}\right)$ forms a group $G$ and a certain subgroup $H$ of $G$ provides us with the $\mathrm{SO}(3)$-bundles we seek.

Before constructing $H$ we need some general results about the construction of bundles. If one wishes to construct $G$-bundles over a compact manifold $M$, then one needs a space $B_{G}$ known as the classifying space for bundles with group $G$. This space $B_{G}$ is the base space of a certain bundle $W_{G}$ called a universal $G$-bundle. Then for a $\operatorname{map} f$

$$
f: M \rightarrow B_{G},
$$

$f^{*} W_{G}$ is a bundle over $M$ known as the pull-back of $W_{G}$ by $f$. All $G$-bundles over $M$ arise as $f^{*} W_{G}$ for some $f$, also if $f$ and $g$ are homotopic maps the $f^{*} W_{G}$ and $g^{*} W_{G}$ are isomorphic. Thus all $G$-bundles over $M$ are given [2] by all homotopy classes of maps $f: M \rightarrow B_{G}$ by $\left[M, B_{G}\right]$. Now we choose $G=\operatorname{SO}(3)$ and $M=T^{4}$ so that we wish to know $\left[T^{4}, B_{\mathrm{SO}(3)}\right]$. Next we may use a result of James et al [3] to characterise $\left[T^{4}, B_{\mathrm{SO}(3)}\right]$ in terms of $\widetilde{K} O\left(T^{4}\right)$. To this end we calculate $\widetilde{K} O\left(T^{4}\right)$. This calculation presents some difficulties which may be circumvented by replacing $T^{4}$ by $X$ where $X$ is a space of the same homotopy type as $T^{4}$ so that $\tilde{K} O\left(T^{4}\right)=\tilde{K} O(X)$. Such a space $X$ is a given by [4]

$$
\begin{array}{rrr}
X=S^{4} v\left(S^{3} v S^{3} v \ldots v S^{3}\right) v\left(S^{2} v S^{2} \ldots v S^{2}\right) v\left(S^{1} v \ldots v S^{1}\right), \\
\text { 4-times } & \text { 6-times } & \text { 4-times }
\end{array}
$$

where $A v B$ denotes the disjoint union of $A$ and $B$ with base points identified. (Alternatively, instead of introducing $X$, one may calculate $\tilde{K} O\left(T^{4}\right)$ via the properties of $\tilde{K} O^{-P}\left(T^{4}\right)$, where $\tilde{K} O^{-P}=\tilde{K} O\left(S^{P} M\right)$ and $S^{P} M$ is the $P$-fold suspension of $M$.) We then have

$$
\begin{array}{r}
\tilde{K} O(X)=\tilde{K} O\left(T^{4}\right)=\tilde{K} O\left(S^{4}\right) \oplus \tilde{K} O\left(S^{3}\right) \oplus \tilde{K} O\left(S^{2}\right) \oplus \tilde{K} O\left(S^{1}\right) . \\
\text { 4-times } \quad \text { 6-times } \quad \text { 4-times }
\end{array}
$$

The right-hand side of 2.3 is well known [2] so that we obtain

$$
\tilde{K} O\left(T^{4}\right)=Z \oplus\left(Z_{2}\right) \text {, }
$$

10-times

where $Z_{2}$ denotes the group of integers modulo 2. Next we utilise Theorem 1.6 of [3] which says that the map

$$
\left[T^{4}, B_{\mathrm{SO}(3)}\right] \rightarrow\left[T^{4}, B_{\mathrm{SO}}\right]=\tilde{K} O\left(T^{4}\right)
$$


is injective, and that under this map the elements of $\left[T^{4}, B_{\mathrm{SO}(3)}\right]$ correspond to a subgroup $H$ of $\tilde{K} O\left(T^{4}\right)$ : namely those elements of $\tilde{K} O\left(T^{4}\right)$ with vanishing 4thStiefel-Whitney class $W_{4}$. (In $2.5 B_{\text {SO }}$ is the classifying space for all principal $\mathrm{SO}(n)$ bundles and SO denotes the infinite special orthogonal group.) The subgroup $H$ is then given by

$$
H=2 \mathbf{Z} \oplus\left(Z_{2}\right) .
$$

6-times

We can now describe the various $\mathrm{SO}(3)$-bundles over $T^{4}$. To do this requires the notion of an induced or pullback bundle: if $f$ is a map from $T^{4}$ to $M$ and $E$ is a bundle over $M$, then $f^{*} E$, the pullback bundle, is a bundle over $T^{4}$. The six $Z_{2}$ summands in $H$ correspond to the following pullbacks. Project first from $T^{4}$ to $T^{2}$ (the 2-torus). This can clearly be done in six possible ways. Denote the six projections by $\pi_{1}, \ldots \pi_{6}$ :

$$
\begin{gathered}
\pi_{i}: T^{4} \rightarrow T^{2} . \\
i=1, \ldots 6
\end{gathered}
$$

Now consider the Hopf bundle $S^{3}$ over $S^{2}$ and a map $f: T^{2} \rightarrow S^{2}$; if we denote the Hopf bundle by $\xi$, then $f^{*} \xi$ is the pullback of $\xi$ to $T^{2}$ and $\left(f \circ \pi_{i}\right)^{*} \xi$ is the pullback to $T^{4}$. These six bundles contain the twist $\eta_{\mu \nu}$ referred to by 't Hooft [1]. They also have zero Pontrjagin number $p_{1}$. This is because

$$
\begin{aligned}
H^{4}\left(S^{2} ; Z\right) & =0 \text { so that } p_{1}(\xi)=0 \text { and } \\
p_{1}\left\{\left(f \circ \pi_{i}\right)^{*} \xi\right\} & =\left(f \circ \pi_{i}\right)^{*} p_{1}(\xi) \\
& =0,
\end{aligned}
$$

so the bundles over $T^{4}$ have vanishing $p_{1}$ also. These bundles $\left(f \circ \pi_{i}\right)^{*} \xi$ are $\operatorname{SO}(3)$ bundles by virtue of the embedding of $\mathrm{U}(1)$, the group of $\xi$, in $\mathrm{SO}(3)$; they correspond to the generators of the six $Z_{2}$ summands in $H$. Further U(1)-bundles may be formed as we shall see below shortly. The summand $2 \mathbf{Z}$ in $H$ is generated by pulling back a certain bundle $\zeta$ over $S^{4}$ to $T^{4}$ under a map $g: T^{4} \rightarrow S^{4}$. The bundle $\zeta$ has total space $\mathbf{C} \mathbf{P}^{3}$ and base space $\mathbf{H} \mathbf{P}^{1}$, where $\mathbf{H} \mathbf{P}^{1}$ stands for one dimensional quaternionic projective space, and in fact $\mathbf{H} \mathbf{P}^{1} \simeq S^{4}$. The fibration is as follows: $\mathbf{C} \mathbf{P}^{3}$ has four homogeneous coordinates $\left[z_{1}, \ldots z_{4}\right]$, a quaternion $q$ may be regarded as being given by a pair of complex numbers $a, b$ so that $q=a+b j$. The projection $p$ of the bundle $\zeta$ projects $\left[z_{1}, \ldots z_{4}\right]$ onto $\left[z_{1}+z_{2} j, z_{3}+z_{4} j\right]$, which is an element of $\mathbf{H P}^{1}$. The pullback $g^{*} \zeta$ is an $\mathrm{SO}(3)$-bundle over $T^{4}$. Further $p_{1}\left(g^{*} \zeta\right)$ is always even. This is because in general we have

$$
p_{1}(\zeta) \bmod 2=W_{2}^{2}(\zeta)
$$

where $W_{2}(\zeta) \in H^{2}\left(S^{4} \mathbf{Z} / 2\right)$ is the second Stiefel-Whitney class of $\zeta$. Since $H^{2}\left(S^{4}: \mathbf{Z} / 2\right)=0$, then $p_{1}(\zeta)$ is even. Now if the map $g: T^{4} \rightarrow S^{4}$ has degree $k$, we have

$$
\begin{aligned}
p_{1}\left(g^{*}(\zeta)\right) & =g^{*} p_{1}(\zeta) \\
& =k p_{1}(\zeta)
\end{aligned}
$$


so that $p_{1}\left(g^{*} \zeta\right)$ is also even, in fact $p_{1}(\zeta)=2$, and thus $p_{1}\left(g^{*} \zeta\right)=2 k$. We have now identified the bundles that correspond to the generators of $H$. The operations of $\otimes$ and $\oplus$ which provide $\tilde{K} O\left(T^{4}\right)$ with its ring structure provide a source of further bundles.

In general we define $\xi_{i}=\left(f \circ \pi_{i}\right)^{*} \xi$ and $\xi_{j}=\left(f \circ \pi_{i}\right)^{*} \xi$; the tensor product $\xi_{i} \otimes \xi_{j}$ remains a $\mathrm{U}(1)$-bundle and will have Pontrjagin number given by, (cf. appendix)

$$
p_{1}\left(\xi_{i} \otimes \xi_{j}\right)=C \varepsilon_{\mu \nu \alpha \beta} \nu^{\mu v} v^{\alpha \beta},
$$

where the integer $\eta^{\mu v}$ is the twist of $\xi_{i} \otimes \xi_{j}$ and $C=\frac{1}{4}$, so that $p_{1}\left(\xi_{i} \otimes \xi_{j}\right)$ is an even integer. Now if we set $\zeta_{i j}=\zeta_{i} \otimes \zeta_{j}$ and form $\tau=\zeta_{i j} \oplus g^{*} \zeta$, then we have, since $H^{4}\left(T^{4} ; \mathbf{Z}\right)$ contains no elements of order 2 ,

$$
\begin{aligned}
p_{1}(\tau) & =p_{1}\left(\zeta_{i j} \oplus g^{*} \zeta\right) \\
& =p_{1}\left(\zeta_{i j}\right)+p_{1}\left(g^{*} \zeta\right) \\
& =2 k+C \varepsilon_{\mu \nu \alpha \beta} \eta^{\mu v} \eta^{\alpha \beta} .
\end{aligned}
$$

Compare this with Eq. (1.1) of ref. 5, cf. also Van Baal [6], where the definition of $p_{1}$ used in ref. 5 is corrected.

\section{The $n>2$ Case}

When $n>2$ the group to be considered is $\mathrm{SU}(n) / Z_{n}$, which we write as $\mathrm{PU}(n)$. Here $\mathrm{PU}(n)$ is the projective unitary group, if $G$ is any group with centre $Z$ then $P G=G / Z$; note that $\mathrm{PU}(n)=\operatorname{PSU}(n)$. The essentials of our problem will again be reduced to the calculation over spheres $S^{i}$ via 2.2; now $G$-bundles over $S^{i}$ are classified by the homotopy group $\pi_{i-1}(G)$, i.e. we have an isomorphism

$$
\left[S^{i}, B_{G}\right] \simeq\left[S^{i-1}, G\right] \text {. }
$$

If $G=\mathrm{SU}(n)$, then it is important to know that

$$
\pi_{i}(\mathrm{PU}(n))=\pi_{i}(\mathrm{SU}(n)), i>1,
$$

but that

$$
\pi_{1}(\mathrm{PU}(n))=Z_{n} ; \pi_{1}(\mathrm{SU}(n))=0 .
$$

We shall refer to a $\mathrm{PU}(n)$-bundle as a projective bundle. Projective bundles may be obtained from $\mathrm{U}(n)$-bundles by a procedure that we now describe, however inequivalent $\mathrm{U}(n)$-bundles may give rise to the same projective bundle: If $E$ is a $\mathrm{U}(n)$ bundle over a manifold $M$, then it gives rise to a projective bundle $P E$ by use of the natural projection $p: \mathrm{U}(n) \rightarrow \mathrm{PU}(n)$. If, however, $L$ is a $\mathrm{U}(1)$-bundle, or line bundle, then $E \otimes L$ is another $U(n)$-bundle; in general inequivalent to $E$, but certainly $P(E \otimes L)=P E$. A converse also holds, i.e. if $P E$ and $P F$ are equivalent projective bundles, then there exists a line bundle $L$ such that

$$
E \simeq F \otimes L \text {. }
$$

There is therefore a one to one correspondence between projective bundles $P E$ and equivalence classes of $\mathrm{U}(n)$-bundles, the equivalence relation is denoted by $\sim$ and 
the equivalence class given by:

$$
E \sim F \Leftrightarrow E=F \otimes L
$$

for some line bundle $L$. To calculate projective bundles $P E$ over $M$ one can therefore first calculate all $\mathrm{U}(n)$-bundles over $M$, and then divide these up into equivalence classes according to (3.4), one then has all the projective bundles $P E$. We have a specific situation: namely $n>2$ and $M=T^{4}$. It is then known that the $\mathrm{U}(n)$-bundles are found by calculating $\tilde{K} U\left(T^{4}\right)$, the reduced complex $K$-theory of $T^{4}$; since $n>2$, we are in what is known as the stable range and two vector bundles of rank $n$ are isomorphic if and only if they are equivalent in $\tilde{K} U\left(T^{4}\right)$. In other words

$$
\left[T^{4}, B_{U(n)}\right] \simeq \tilde{K} U\left(T^{4}\right), \quad n>2 .
$$

The calculation of $\tilde{K} U\left(T^{4}\right)$ is done by exactly similar methods to those used in Sect. 2 and the result is

$$
\begin{aligned}
K U\left(T^{4}\right)= & K U\left(S^{4}\right) \oplus K U\left(S^{3}\right) \oplus K U\left(S^{2}\right) \oplus K U\left(S^{1}\right) \\
& \text { 4-times } 6 \text {-times } 4 \text {-times } \\
= & \mathbf{Z} \oplus \mathbf{Z} \oplus \ldots \mathbf{Z} .
\end{aligned}
$$

In the right-hand side of (3.6), one copy of $\mathbf{Z}$ comes from the fact that $\widetilde{K} U\left(S^{4}\right)=\mathbf{Z}$, the other six come from the fact that $\widetilde{K} U\left(S^{2}\right)=\mathbf{Z}, \widetilde{K} U\left(S^{3}\right)$ and $\widetilde{K} U\left(S^{1}\right)$ being zero. The description of the $\mathrm{U}(n)$-bundles over $T^{4}$ requires first the giving of the bundles over $S^{2}$ and $S^{4}$ that correspond to the generators of $K U\left(S^{2}\right)$ and $K U\left(S^{4}\right)$. We denote these bundles by $\xi$ and $\zeta$ respectively, $\xi$ is determined by a map

$$
\alpha: S^{1} \rightarrow \mathrm{U}(n)
$$

and $\zeta$ by a map

$$
\beta: S^{3} \rightarrow \mathrm{U}(n) .
$$

In fact $\alpha \in \pi_{1}(\mathrm{U}(n))=\mathbf{Z}$ and $\beta \in \pi_{3}(\mathrm{U}(n))=\mathbf{Z}$ so that only the homotopy classes of $\alpha$ and $\beta$ matter. The integers, $a$ and $b$ say, that label the homotopy class of $\alpha$ and $\beta$ respectively are chosen to be unity and are given by

$$
\begin{aligned}
& a=\int_{S_{2}} C_{1}(\xi), \\
& b=\int_{S_{4}} C_{2}(\zeta),
\end{aligned}
$$

where $C_{1}$ and $C_{2}$ denote Chern classes. To construct $\mathrm{U}(n)$-bundles over $T^{4}$ we simply need to pull-back $\xi$ and $\zeta$ to $T^{4}$, i.e. to construct the bundles $\left(f \circ \pi_{i}\right)^{*} \xi$ and $g^{*} \zeta$.

Finally we need to construct $\mathrm{PU}(n)$-bundles over $T^{4}$. This is done by giving $P \xi$ and $P \zeta$ : let $p$ be the projection $p: \mathrm{U}(n) \rightarrow \mathrm{PU}(n)$, then the maps

$$
\begin{aligned}
& \alpha_{p}: S^{1} \stackrel{\alpha}{\rightarrow} \mathrm{U}(n) \stackrel{p}{\rightarrow} \mathrm{PU}(n), \\
& \beta_{p}: S^{3} \stackrel{\beta}{\rightarrow} \mathrm{U}(n) \stackrel{p}{\rightarrow} \mathrm{PU}(n),
\end{aligned}
$$

where $\alpha_{p}=p \circ \alpha$ and $\beta_{p}=p \circ \beta$ define the projective bundles $P \xi$ and $P \zeta$ which are then 
pulled back to $\left(f \circ \pi_{i}\right)^{*} P \xi$ and $g^{*} P \zeta$ respectively to give projective bundles over $T^{4}$. Evidently $\alpha_{p} \in \pi_{1}(\mathrm{PU}(n))=Z_{n}$ and $\beta_{p} \in \pi_{3}(\mathrm{PU}(n))=\mathbf{Z}$. Thus the six kinds of bundles $\left(f \circ \pi_{i}\right)^{*} P \xi$ are classified by a twist $\eta_{\mu \nu}$ defined modulo $n$ and the bundles $g^{*} P \zeta$ are classified by an integer. The twist $\eta_{\mu \nu}$ is defined modulo $n$ because $\alpha \in \pi_{1}(\mathrm{U}(n))=\mathbf{Z}$ and $\alpha_{p} \in \pi_{1}(\mathrm{PU}(n))=Z_{n}$, for example, because of this fact, two homotopically inequivalent $\alpha, \alpha^{\prime}: S^{1} \rightarrow \mathrm{U}(n)$ may become homotopically equivalent when composed with $p$, i.e. we may have $\alpha \neq \alpha^{\prime}$ but $\alpha_{p} \simeq \alpha_{p}^{\prime}$.

In fact since topologically $\mathrm{U}(n)=\mathrm{U}(1) \times \mathrm{SU}(n)$, then $\pi_{1}(\mathrm{U}(n))=\pi_{1}(\mathrm{U}(1))$, since $\pi_{1}(\mathrm{SU}(n))=0$, so that in (3.10) the map $\alpha \in \pi_{1}(\mathrm{U}(n))$ is actually determined by an element $\alpha^{\prime}$ of $\pi_{1}(\mathrm{U}(1))=\pi_{1}\left(S^{\prime}\right)$ and $\operatorname{deg} \alpha^{\prime}$, the degree of $\alpha^{\prime}$, is unity. This means that $\xi$ is again the Hopf bundle of Sect. 2. As a consequence we may again construct the $\operatorname{PU}(n)$-bundle $\tau$ where

$$
\tau=\zeta_{i j} \oplus g^{*} P \zeta
$$

and $\zeta_{i j}=\xi_{i} \otimes \xi_{j}$ and $\xi_{i}=\left(f \circ \pi_{i}\right)^{*} P \xi, \xi_{j}=\left(f^{\prime} \circ \pi_{j}\right)^{*} P \xi$. Even though $\tau$ was originally derived from $\mathrm{U}(n)$-bundles whose characteristic classes are Chern classes, $\tau$ may be regarded as having a Pontrjagin class $p_{1}(\tau)$. This point, also made independently by Van Baal [6], is that PU(n) is isomorphic to a subgroup $G$ of $\mathrm{SO}\left(n^{2}-1\right)$, indeed any compact Lie group is isomorphic to a subgroup of $\mathrm{O}(n)$ for some $n$. The isomorphism in the case of $\mathrm{PU}(n)$ is provided by simply taking the adjoint representation of $\mathrm{U}(n)$, the map defining the adjoint representation has, by definition, kernel equal to the centre of $\mathrm{U}(n)$ so that the desired isomorphism $\mathrm{PU}(n) \simeq \operatorname{AdU}(n)$ follows. This being so, a $\mathrm{PU}(n)$-bundle may be regarded as an $\mathrm{SO}\left(n^{2}-1\right)$-bundle whose structure group reduces to $G$, its appropriate characteristic class can then be taken to be a Pontrjagin class.

A general Abelian configuration is given by taking a sum of $(n-1)$-bundles $\zeta_{i j}$ which we denote by $\zeta^{(a)}, a=1, \ldots n-1$. The resulting bundle, $\zeta$ say, has group $\mathrm{SO}(2) \times \ldots \times \mathrm{SO}(2),((n-1)$-times $)$, which corresponds to the maximal Abelian subalgebra for $\operatorname{AdU}(n) \subset \mathrm{SO}\left(n^{2}-1\right), n>2$. For $\zeta$ we have

$$
\begin{aligned}
p_{1}(\zeta) & =p_{1}\left(\zeta^{(1)} \oplus \zeta^{(2)} \ldots \oplus \zeta^{(n-1)}\right) \\
& =p_{1}\left(\zeta^{(1)}\right)+\ldots p_{1}\left(\zeta^{(n-1)}\right) \\
& =\sum_{a=1}^{n-1} \frac{\varepsilon_{\mu v \alpha \beta}}{4} \hat{\eta}_{\mu \nu}^{(a)} \hat{\eta}_{\alpha \beta}^{(a)}
\end{aligned}
$$

These $\hat{\eta}_{\mu \nu}^{(\alpha)}$ differ from those of ref. 6 due to a difference in the normalisation of the subalgebra. With the normalisation of ref. 6 we indeed find

$$
p_{1}(\zeta)=\frac{(n-1)}{4} \varepsilon_{\mu \nu \alpha \beta} \eta_{\mu \nu} \eta_{\alpha \beta}+k,
$$

where $\eta_{\mu \nu}$ is the twist defined modulo $n$ and $k$ is an even integer. The splitting principle [2] guarantees that a general value of $p_{1}$ may be obtained with such Abelian configurations in agreement with refs. 1 and 6, and $p_{1}$ is also always even [6]. 


\section{Appendix}

Let the bundle $\xi_{i}$ have twist $\eta_{\mu v}$ and $\xi_{j}$ have twist $\eta_{\alpha \beta}$ as U(1)-bundles. Then we have

$$
\begin{aligned}
& c_{1}\left(\xi_{i}\right)=\eta_{\mu \nu}=\int_{T^{2}} i \frac{\mathbf{F}^{i}}{2 \pi}, \\
& c_{2}\left(\xi_{j}\right)=\eta_{\alpha \beta}=\int_{T^{2}} i \frac{\mathbf{F}^{j}}{2 \pi},
\end{aligned}
$$

where $\mathbf{F}^{i}$ and $\mathbf{F}^{j}$ are curvature defined on 2-dimensional tori. The Pontrjagin class $p_{1}\left(\xi_{i} \otimes \xi_{j}\right)$ is given by [2]

$$
\begin{gathered}
p_{1}\left(\xi_{i} \otimes \xi_{i}\right)=\left\{c_{1}\left(\xi_{i} \otimes \xi_{j}\right)\right\}^{2}=\left\{c_{1}\left(\xi_{i}\right)+c_{1}\left(\xi_{j}\right)\right\}^{2}, \\
c_{1}^{2}\left(\xi_{i}\right)+c_{1}\left(\xi_{i}\right) c_{1}\left(\xi_{j}\right)+c_{1}\left(\xi_{j}\right) c_{1}\left(\xi_{i}\right)+c_{1}^{2}\left(\xi_{j}\right)=2 c_{1}\left(\xi_{i}\right) c_{1}\left(\xi_{j}\right),
\end{gathered}
$$

where we have used the facts that $c_{1}^{2}\left(\xi_{i}\right)=c_{1}^{2}\left(\xi_{j}\right)=0$ and $c_{1}\left(\xi_{i}\right) c_{1}\left(\xi_{j}\right)=c_{1}\left(\xi_{j}\right) c_{1}\left(\xi_{i}\right)$, which follow from naturality and $\mathrm{U}(1)$-valuedness respectively.

Thus

$$
p_{1}\left(\xi_{i} \otimes \xi_{j}\right)=-\frac{2}{(2 \pi)^{2}} \int_{T^{4}}\left(\pi_{i}^{*} \mathbf{F}^{i}\right) \wedge\left(\pi_{j}^{*} \mathbf{F}^{j}\right),
$$

where $\pi_{i}^{*} \mathbf{F}^{i}$ and $\pi_{j}^{*} \mathbf{F}^{j}$ are the pullbacks of the curvatures $\mathbf{F}^{i}$ and $\mathbf{F}^{j}$ to $T^{4}$. The righthand side of (A.3) is evidently proportional to $\varepsilon_{\mu \nu \alpha \beta} \eta_{\mu \nu} \eta_{\alpha \beta}$. If one takes a specific case where $\eta_{\mu v}=\eta_{12}, \eta_{\alpha \beta} \equiv \eta_{34}$, one finds easily that this constant is $\frac{1}{4}$, so we have

$$
p_{1}\left(\xi_{i} \otimes \xi_{j}\right)=\frac{1}{4} \varepsilon_{\mu \nu \alpha \beta} \eta_{\mu \nu} \eta_{\alpha \beta}
$$

as desired, and this formula holds for general $\eta_{\mu \nu}, \eta_{\alpha \beta}$ defined modulo $n$.

Acknowledgements. The author would like to thank Richard Ward for his encouragement and Professor I. James for a valuable correspondence.

\section{References}

1. Ambjфrn, J., Flyvbjerg, H. :'t Hooft's non-Abelian magnetic flux has zero classical energy. Phys. Lett. 97B, 24 I (1980); 't Hooft, G.: A property of electric and magnetic flux in non-Abelian gauge theories. Nucl. Phys. B153, 141 (1979); Physica Scripta 24, 841 (1981): Acta Phys. Aust. Suppl. 52, 531 (1980); Some twisted self-dual solutions for the Yang-Mills equations on a hypertorus. Commun. Math. Phys. 81, 267 (1981)

2. Eguchi, T., Gilkey, P. B., Hanson, A. J.: Gravitation, gauge theories and differential geometry. Phys. Rep. 66, 213 (1980); Husemdler, D.: Fibre bundles. Berlin-Heidelberg, New York: Springer 1975; Atiyah, M. F.: K-theory. New York: Benjamin Inc. 1967.

3. James, I., Thomas E.: J. Math. Mech, 14, 485 (1965) (I am indebted to Professor I. James for this information.)

4. James, I.: Trans. Am. Math. Soc. 84. 545 (1957)

5. 't Hooft, G.: Some twisted self-dual solutions for the Yang-Mills equations on a hypertorus. Commun. Math. Phys. 81, 267 (1981)

6. Van Baal, P.: Utrecht preprint

Communicated by R. Stora

Received February 25, 1982; in revised form June 3, 1982 
